\title{
Research on three dimensional simulation method for building based on 3D dynamic vision
}

\author{
Wang Li \\ Tianjin Construction Engineering Staff and Workers University, 300074
}

Keywords: perspective drawing; design sketch; 3D modeling

\begin{abstract}
Perspective reverse is applied to the design sketch of building with the basic principle of perspective drawing and application data fusion algorithm, the perspective condition is determined by analysis and calculation, so as to realize the transformation from two-dimensional information (2D) to three-dimensional (3D) information, and achieve 3D reconstruction. In 3D modeling process, the future building reflected by architecture perspective drawing should be not only consistent with design sketch, but also composing and rendering from the perspective of art. Because the computer is easy to produce perspective distortion during the 3D simulation modeling process, it is necessary to study the effect of perspective distortion on vision -- Perspective effect. Thus, the distortion in 3D modeling process is reduced, the reality of 3D modeling is improved.
\end{abstract}

\section{Introduction}

With the development of society and the progress of technology, people have higher demand on the architectural form, therefore, in order to have the stable development of the sales market, the construction enterprise must put building design in an important position, so that the form of architecture meet the needs of the market [1-3]. And the primary stage of architectural design are mostly designing 2D drawings by the designer, architectural design 2D drawings can give full play to the space imagination of designer, it is a record of the process, the result formed is design sketch [4-6].

Although design sketch reflects the effect of the design of the building quickly and efficiently, but it is still a 2D drawing, for applying in construction, according to the design mode of modernization, 3D reconstruction is necessary for the drawing[7,8]. At present, 2D graphics or images are directly used to obtain the 3D data, and then establish the three-dimensional model, this method has made progress, but the method of stereo vision is used more often, in fact, it is reverse engineering based on a real object. In this paper, the 3D reconstruction based on the basic principles of perspective drawing and design sketch, according to some geometric properties in the sketch to seek perspective conditions, so as to realize the $3 \mathrm{D}$ reconstruction $[9,10]$.

\section{Perspective Conditions in Architectural Perspective Drawing}

Architectural perspective drawing is projection graph obtained by the method of the center projection, this perspective drawing have strong three-dimensional sense and distinctive building features, so it has been widely applied. In the perspective view, according to the degree of inclination of buildings and the perspective picture in perspective imaging, the perspective drawing can be divided into one point, two point and three point perspective. Because the two point perspective can avoids distortion of perspective effect to the maximum, and express the design intention better, it is a perspective drawing of the most commonly used, so this paper mainly discusses the situation of two point perspective. The principle of two point perspective formation and condition as shown in Figure 1. 


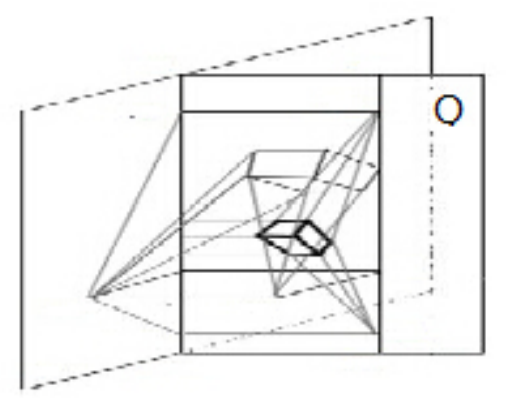

Figure 1 the formation principle and perspective conditions of two point perspective drawing

The graph composed of the thick solid line in the figure 1 is two point perspective drawing of the spatial objects on the picture Q, the so-called perspective conditions are the parameter referring to the mutual position relationship of view point, pictures and architectures, thus completing the implementation process of 3D reconstruction.

\section{1 basic steps of $3 D$ reconstruction}

According to the structure characteristics of the architectural form, firstly, it can be split into a plurality of geometric shapes, the rectangular bounding box (BOX) is utilized to contain various geometric body parts, and then calculating the perspective condition, so as to process composition correction according to the ratio of various parts.

1.2 analysis of architectural perspective drawing, determine the perspective conditions

In order to calculate the architectural appearance accurately and reliably, precise perspective conditions must be found in view of the design sketch. When drawing the perspective, the perspective condition have to be determined to do layout for perspective, then the drawing of architecture perspective can be started, the reverse perspective of building is as well. Based on the basic principle and data fusion algorithm of architecture perspective, firstly, find the vanishing points, and then on the basis of the vanishing point combined with perspective selection principle to determine the other perspective conditions, such as site, perspective angle etc..

22.1 reverse method for geometric elements in perspective of construction

The reverse method is the inverse process of drawing architecture perspective, which is based on architectural perspective to obtain 3D data model of construction reversely. The main methods include measuring point method, line of sight method, across the board intersection method and distance point method.

First, according to the perspective principle of parallel lines to find the two vanishing points, and then calculating the perspective conditions, so that the projection of architecture can be acquired reversely by measuring point method.

2 2.2 Reverse method for perspective conditions, like vanishing points and the site

According to the principles of architectural perspective, a set of parallel lines have only one vanishing point. Extending the parallel lines, the intersection point is their vanishing point. With this principle can find all the vanishing point. However, the sketch is nor normative, there will be multiple intersection points. The following discussion is about the overall BOX of some architectural design sketch, the vanishing point of two direction are respectively G1, G2, BOX vanishing points of the geometry of each part are as shown in the following figure, clearly has multiple locations.

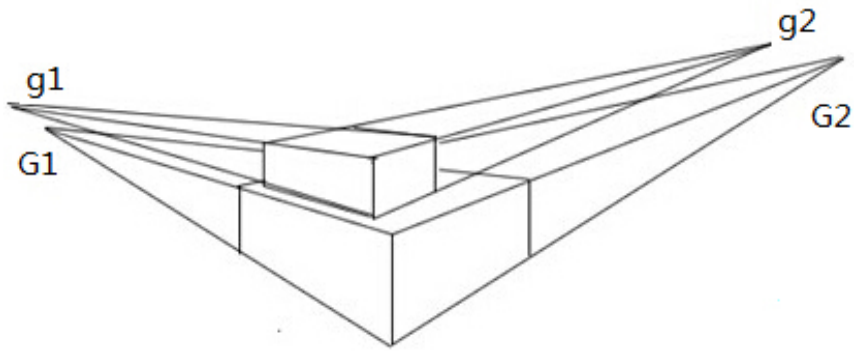

Figure 2 BOX vanishing points 
According to the above, a comprehensive treatment have to be applied to the multiple points, and ultimately find a suitable point, on the basis of confidence distance, data fusion algorithm of the degree of mutual support between data is utilized in this paper to process the data, so as to seek G1 and G2, and achieve 3D simulation of the building.

\section{Perspective Distortion Compensation}

On the one hand, the designer must have the correct visual perception and visual expression on perspective of building. Besides, for the optical lens, the lens quality has a direct impact on architectural image quality. As for the optical lens of high quality, when focal length is less than 18 $\mathrm{mm}$, the image was a barrel (optical distortion). Since zoom function buttons included in 3D MAX and VIZ, disturbing factors described earlier has been ruled out during the design stage of software, so within the range of focal length of the lens, the optical distortion is not shown in the perspective sketch in computer. The model established by 3D MAX or VIZ, with objective lenses - Select Target key, as the lens and target icon are regarded as standard objects in 3DMAX, the camera view can be employed as a wizard. In the 3D space, standard transformation is utilized for precisely positioning lens, with Perspective converted into Camera to observe 3D graph, shown in Figure 3.

\section{1 viewpoint and perspective of the relationship}

When the distance between viewpoint $\mathrm{P}$ and the wall BC is large (solid line in FIG. 4), the graph becomes smaller; $\mathrm{BP} / \mathrm{CP}=19 \mathrm{~m} / 10 \mathrm{~m}=1.9: 1$, C'

$\mathrm{C}^{\prime \prime} / \mathrm{B}^{\prime} \mathrm{B} "=1.9: 1$, the ratio is same to $\mathrm{BP} / \mathrm{CP}$, as the ratio is smaller, the slope is decreased. Its visual performance is small perspective space in the $\mathrm{X}$-axis, and strong visual compression, walls $\mathrm{B}$, $\mathrm{C}$ have visual compression in the $\mathrm{Y}$-axis. As the distance between the viewpoint $\mathrm{P}$ and $\mathrm{BC}$ wall decreases (broken line in FIG. FIG. 4), BP / CP $=12 \mathrm{~m} / 3 \mathrm{~m}=4: 1$, the larger the ratio, the slope is larger. Similarly $C^{\prime} C$ "/ B'B" $=4: 1$, the graphics near to the point $\mathrm{P}$ is larger and produce a greater distortion. $\mathrm{B}, \mathrm{C}$ was elongated visually in the $\mathrm{X}$-axis, also elongated in the $\mathrm{Y}$-axis. Short distance, short focal length, is exaggerated (distortion) perspective. By exaggerating the ratio to highlight the main building, this principle can be adopted to manufactured dynamic angle of building and exaggerate their height.

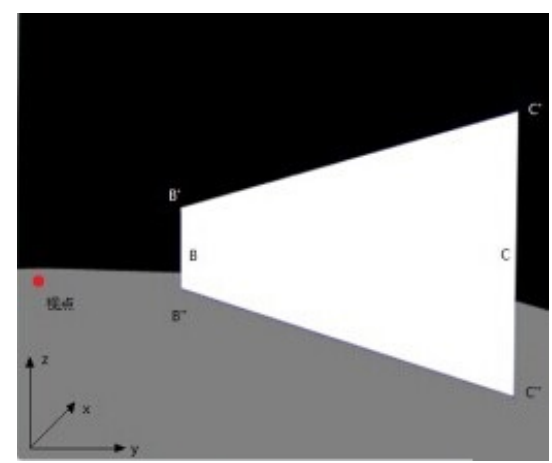

Figure 3 front view

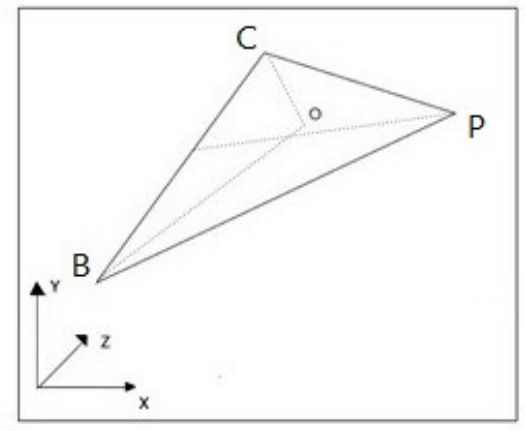

Figure 4 top view

In Figure 3, due to the movement of viewpoint, the image size and slope are changed. Target icon does not move, the shorter visual distance, convergence of horizontal lines of tilt wall is more obvious, the greater the distortion, the more it seem exaggerated, which could easily lead to C ', C 
"terminal overflow from the frame. So the focal length is changed while changing the line of sight, will impact perspective of the screen significantly.

\section{2 relationship of focal length, vision distance and visual horizon}

Each focal length value pattern of short distance is added up (Fig. 5); when the viewpoint is moved farther, since the graphic is too small, similarly, each focal length value pattern in the picture is amplified proportional to original picture and superimposed in PHOTOSHOP, such that each B'B " are same (Fig. 6), whereby the relationship between the respective patterns are compared and analyzed.

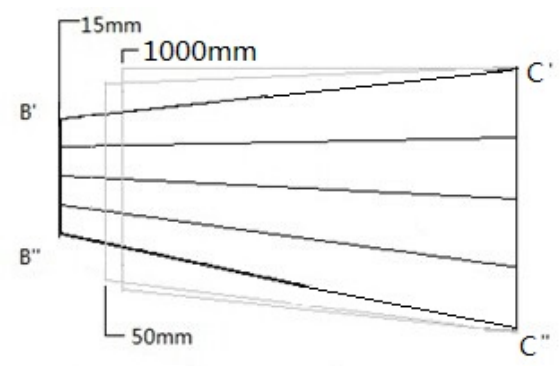

Figure 5 superposition of each focal length graphic with short horizon

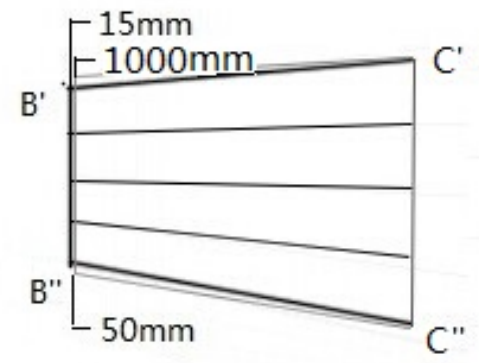

Figure 6 amplification and superposition of each focal length graphic with short horizon

1. When the focal length is $15 \mathrm{~mm}$, a viewpoint from near to farther, as shown in figure 5,6 . As it can be seen from Figure 5, the slope of graphics is high, serious distortion happens to graphics, depth of perspective performance is big, and the graph is elongated visually.

2. When the focal length is $50 \mathrm{~mm}$, perspective relationships of building image is real. In computer graphics, perspective renderings transformed from 3D (Composition) into 2D are same to the photos. At this time, the graphics of viewpoint from near to far are as figure 5, 6 .

3. When the focal length is $1000 \mathrm{~mm}$, which $\mathrm{B}, \mathrm{C}$ have fixed length, $\mathrm{C}^{\prime} \mathrm{C}$ "/ B'B" ratio close to 1 , the reverse distortion increases. Modeling in the VIZ, 3D MAX, camera (the same viewpoint and line of sight) with focal length of $1000 \mathrm{~mm}$ and $50 \mathrm{~mm}$ to get two figures. The graphics of $50 \mathrm{~mm}$ focal length is zoomed in PHOTOSHOP and made into translucent layers, and then fine-tune to make superposition with the graphics of $1000 \mathrm{~mm}$ focal length (Figure 7), which proved two superimposed graphics are completely overlapped.

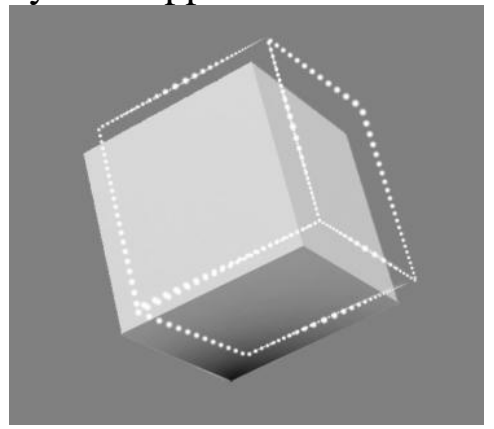

Figure 7 superposition

Similarly, the same experiment is done for real building with camera and obtain the same results. Therefore, whether VIZ, 3D MAX or cameras, when viewpoint, sight distance (object distance) unchanged, change the focal length, its perspective point stays constant. 


\section{Conclusions}

This paper describes a method for the three-dimensional modeling of the building. Perspective reverse is applied to the design sketch of building with the basic principle of perspective drawing and application data fusion algorithm, the perspective condition is determined by analysis and calculation, so as to realize the transformation from two-dimensional information to threedimensional (3D) information, and achieve 3D reconstruction. In 3D modeling process, the future building reflected by architecture perspective drawing should be not only consistent with design sketch, but also composing and rendering from the perspective of art. Because the computer is easy to produce perspective distortion during the 3D simulation modeling process, it is necessary to study the effect of perspective distortion on vision -- Perspective effect. Thus, the distortion in 3D modeling process is reduced, the reality of 3D modeling is improved.

\section{References}

[1] Li Zhentao, Xu Miaozhong. Manufacturing technology for three dimensional perspective of large wooden buildings [J]. Huazhong architecture, 2008.6:58-59.

[2] Zhang Yushuang, Wang Ziru. Light plane cutting method and its application in the blanking of perspective [J]. Journal of Dalian University of Technology, 2001.3:334-338.

[3] Chen Xin, Yang Wanjian. Lighting processing of outdoor architectural renderings [J]. Journal of Guangzhou University: Natural Science Edition, 2003.3:260-263.

[4] Wu Mingyun. Building Perspective renderings. The perspective effect of Architectural Renderings [J]. Journal of Fujian Agriculture and Forestry University: Philosophy and Social Sciences Edition, 2005.3:90-92.

[5] Wei Chuliang, Luo Peiyu, Hong Xiaobin, Qiu Chaole, Cai Zemin. Research on the new method of three image reconstruction based on the model of single pictures [J]. Chinese testing, 2014, 40 (2): 23-26.

[6]Qiao Huiping. Application of image processing software in architectural renderings later processing [J]. Shanxi architecture, 2007.36:367-368.

[7] Yang Wei, Gu Ming. Numerical simulation on the three-dimensional unsteady wind field of high-rise building [J]. Journal of Tongji University: Natural Science Edition, 2003.6:647-651.

[8] Deng Fei, Zhang Zuxun, Zhang Jianqing. Research on three-dimensional reconstruction of ancient buildings using laser scanning and digital cameras [J]. Science of Surveying and Mapping, 2007.2:29-30.

[9] Wang Mingjun, Zhu Wenyao. Research and simulation on 3D virtual reconstruction method for ancient architecture [J]. Computer simulation 2014.3:437-440.

[10] Zhou Yueting, Lu Lingyi. Numerical simulation of three-dimensional unsteady wind field parallel computation in high-rise building [J]. Journal of architecture science and engineering, 2007.1:42-46. 